\title{
Reflexões e Desafios sobre a Formação na Ética em Pesquisa na Computação envolvendo Humanos
}

\author{
Esdras L. Bispo Jr. ${ }^{1,2}$, Liliane S. S. Fonseca ${ }^{3}$, Simone C. Santos ${ }^{2}$ \\ ${ }^{1}$ Universidade Federal de Jataí (UFJ) \\ ${ }^{2}$ Centro de Informática (CIn) - Universidade Federal de Pernambuco (UFPE) \\ ${ }^{3}$ Universidade Católica de Pernambuco (UNICAP) \\ bispojr@ufj.edu.br, liliane.fonseca@unicap.br, scs@cin.ufpe.br
}

\begin{abstract}
This essay aims to bring reflections and challenges about the formation of research ethics in Computing involving humans. The subject is presented as in a general context as in the specific context of Computing.
\end{abstract}

Resumo. Este ensaio tem como objetivo trazer reflexões e desafios sobre a formação na ética em pesquisa na Computação envolvendo humanos. $O$ tema é apresentado tanto no contexto geral, como no contexto específico da Computação.

\section{Introdução}

A ética em pesquisa na Computação envolve vários atores como os sujeitos da pesquisa, os pesquisadores responsáveis, as instituições realizadoras/apoiadoras e os órgãos de fomento. Ela pode ser enxergada como um objeto de estudo da metodologia de pesquisa científica na Computação [Wazlawick 2014]; ou como um objeto da Ética em Computação de uma forma geral [Tedre 2007].

Dentre as várias questões abordadas na ética em pesquisa na Computação, a pesquisa envolvendo seres humanos dispara várias discussões e considerações. Pode-se dar exemplos como os aspectos relacionados à obtenção do consentimento livre e esclarecido do participante e a garantia da confidencialidade dos seus dados que podem ser eventualmente coletados. Preservar as condições necessárias para que os avanços nas pesquisas científicas ocorram sem ferir à dignidade e os direitos humanos historicamente reconhecidos é um dos desafios da ética em pesquisa na Computação.

Embora os códigos de ética profissionais sinalizem para questões importantes, eles não abordam situações específicas enfrentadas pelo pesquisador na Computação. Esses códigos de ética, tais como o ACM Code of Ethics 1 e o Código de Ética da SBC? são focados em questões éticas que emergem originalmente da prática do profissional da Computação [Singer e Vinson 2002, Masiero 2000]. A ética em pesquisa na Computação carece de uma maior reflexão e aprofundamento de suas discussões, principalmente no que diz respeito à formação dos pesquisadores.

Buchanan e colegas apresentaram a formação inicial de pesquisadores na Computação como um dos desafios para a área [Buchanan et al. 2011, p. 79]. Para os autores,

\footnotetext{
${ }^{1}$ Disponível em https: //www.acm.org/code-of-ethics.

${ }^{2}$ Disponível em https://www.sbc.org.br/institucional-3/codigo-de-etica.
} 
pesquisadores em Computação que são expostos a questões sobre ética em pesquisa envolvendo humanos durante sua formação inicial desenvolverão melhores práticas de pesquisa no futuro.

Na mesma direção, Vinson e Singer afirmam que para o pesquisador se comportar eticamente, é necessário que ele compreenda quais são os princípios éticos subjacentes que estão envolvidos [Vinson e Singer 2008, p. 230]. Para isso, os autores defendem que esses princípios sejam devidamente desenvolvidos na formação inicial dos pesquisadores na Computação [Vinson e Singer 2008, p. 254].

Assim, este ensaio tem como objetivo trazer algumas reflexões e desafios sobre a formação na ética em pesquisa na Computação envolvendo humanos. O tema é apresentado tanto no contexto geral, como no contexto específico da Computação.

O restante do ensaio é dividido como se segue. A Seção 2 traz uma reflexão sobre a importância e a relevância da ética em pesquisa na Computação. A Seção 3 apresenta uma segunda reflexão, resgatando alguns momentos históricos importantes da ética em pesquisa envolvendo humanos no Brasil. A Seção 4 elenca os grandes quatro princípios que a ética em pesquisa envolvendo humanos está alicerçada, com um enfoque na Computação. A Seção 5 propõe vários desafios existentes da formação na ética em pesquisa na Computação envolvendo humanos. E, por fim, a Seção6traz as considerações finais do ensaio.

\section{Importância e Relevância da Ética em Pesquisa na Computação}

Uma primeira reflexão sobre a formação na ética em pesquisa na Computação envolvendo humanos diz respeito à importância e à relevância da ética em pesquisa. A primeira seção diz respeito à importância da ética em pesquisa, de uma forma geral, na perspectiva do pesquisador (Seção 2.1). A segunda seção refere-se à relevância da investigação científica da ética em pesquisa na Computação (Seção 2.2).

\subsection{Importância da ética em pesquisa na perspectiva do pesquisador}

A ética cumpre uma função crucial na concepção, planejamento e execução de uma pesquisa científica. Desconsiderar a sua importância pode aumentar os riscos de insucesso na pesquisa, levando até a resultados indesejados.

Uma primeira consequência indesejada diz respeito à integridade subjetiva do pesquisador. Ignorar a discussão sobre ética em pesquisa pode levar a conflitos de integridade em um nível intrapessoal [Noelliste 2013, p. 490]. A não compreensão correta sobre as razões de existir das boas práticas em pesquisa pode promover uma fragmentação interna do pesquisador em relação às exigências externas propostas pela comunidade científica. A discussão sobre a natureza desses conflitos pode contribuir para que o pesquisador realize a sua pesquisa de uma forma mais integral.

Uma segunda consequência indesejada é a possível falta de cooperação e honestidade dos participantes da pesquisa [Singer e Vinson 2002, p. 1171]. Se os participantes sentirem-se coagidos a realizar determinada atividade de pesquisa, o sentimento de inadequação pode se sobrepor ao sentimento de acolhimento e voluntarismo. Assim, pode ocorrer de os participantes reagirem negativamente à situação proposta pelos pesquisadores responsáveis, não cooperando ativamente com a pesquisa ou, em último caso, sendo desonestos com a veracidade das informações fornecidas. 
Uma outra consequência possível é a descredibilização da pesquisa por órgãos de fomento à pesquisa. O Conselho Nacional de Desenvolvimento Científico e Tecnológico (CNPq) e a Fundação de Amparo à Pesquisa do Estado de São Paulo (FAPESP), por exemplo, mantêm políticas explícitas sobre os princípios éticos que o pesquisador deve perseguir ao conduzir a sua pesquisa. O Código de Boas Práticas Científicas ${ }^{3}$ da FAPESP e a Comissão de Integridade na Atividade Científica (CIAC) ${ }^{4}$ do CNPq são iniciativas que têm por objetivo educar pesquisadores sobre integridade em pesquisa e a adoção de boas práticas; e, eventualmente, propor ações cabíveis em caso de má conduta na execução ou publicação dos resultados das pesquisas.

E, por fim, uma última consequência indesejável é a perda da credibilidade do cientista diante da comunidade científica e do seu ecossistema de pesquisa. É desejável que o pesquisador submeta a sua pesquisa à crítica e ao escrutínio de seus pares [Rallis e Lawrence 2017, p. 35]. A validação dos resultados supostamente obtidos pelo pesquisador pode ser questionada pela comunidade científica. Naturalmente, a incidência recorrente de deslizes éticos na condução de atividades científicas prejudica a credibilidade do pesquisador diante de sua comunidade e de organizações interessadas no resultado da pesquisa.

\subsection{Relevância da ética em pesquisa na Computação}

Pode-se fazer um recorte na ética em pesquisa, delimitando-a a partir de suas relações com a Ciência da Computação. A ética em pesquisa pode ser vista como uma área da Filosofia da Computação [Brey e Søraker 2009, p. 1390], tendo relevância científica específica e lacunas de pesquisas importantes a serem exploradas.

Há uma quantidade expressiva de trabalhos na área da ética profissional, como [Johnson 2008, Kizza 2016]. Entretanto, há uma carência de trabalhos na ética em pesquisa na Computação, principalmente no que diz respeito aos aspectos da pesquisa envolvendo humanos [Singer e Vinson 2002, p. 1172].

É importante salientar que algumas áreas da Computação estão mais preocupadas com esses aspectos da pesquisa envolvendo humanos. Esta realidade é mais comum em áreas como Educação em Computação (EC), Engenharia de Software Empírica (EEE), Informática na Educação (IE) e Interação Humano-Máquina (IHC). Essas áreas adotam métodos empíricos de pesquisa que são largamente utilizados em outros campos de conhecimento como Psicologia e Sociologia [Easterbrook et al. 2008, p. 285]. Esse é um dos motivos que pode justificar a ausência de investigações mais aprofundadas com este recorte.

Vários aspectos da ética em pesquisa envolvendo humanos são compartilhados por diversos campos do conhecimento. Entretanto existem alguns recortes de investigação que emergem exclusivamente dentro da pesquisa na Computação. É necessário, por exemplo, que os estudantes de uma graduação em Computação aprendam diversos paradigmas de linguagem de programação durante a sua formação. A partir desse aspecto, existem pesquisas investigando qual seria a melhor estruturação curricular: Deveria ser ministrada primeiro programação procedural ou programação orientada a objetos? [Cooper et al. 2003, Kunkle e Allen 2016]. Investigar esse objeto exige do pesquisador

\footnotetext{
${ }^{3}$ Ver em http: //www. fapesp.br/boaspraticas/

${ }^{4}$ Ver em http://gg.gg/cnpq-comissao-integridade.
} 
algumas tomadas de decisão importantes que poderão afetar significativamente a qualidade da formação do estudante/participante da pesquisa. Diante disso, seria eticamente correto dividir a mesma turma em dois grupos, sendo um de controle e outro de teste, de forma que um grupo esteja submetido a uma estruturação curricular e o outro não?

Um outro recorte de investigação, que emerge dentro da ética em pesquisa na Computação envolvendo humanos, é sobre os aspectos relacionados à formação de pesquisadores sobre esse tema. Há trabalhos desenvolvendo questões sobre o ensino de filosofia da Computação [Stahl 2019], mas há uma carência de trabalhos em relação à formação desses pesquisadores. Existem até referências a essa formação de forma pulverizada em disciplinas da Computação [Saltz et al. 2019], porém sem uma intencionalidade clara e integrada.

\section{3. Ética em pesquisa envolvendo humanos no Brasil}

Uma segunda reflexão sobre a formação na ética em pesquisa na Computação envolvendo humanos diz respeito à evolução da ética em pesquisa no Brasil. Compreender essa evolução permite que os docentes e pesquisadores da Computação se percebam como parte da construção e do amadurecimento da pesquisa em nosso país.

No Brasil, a ética em pesquisa envolvendo humanos é referendada pela Comissão Nacional de Ética em Pesquisa (CONEP). A CONEP é ligada ao Conselho Nacional de Saúde (CNS) e tem como principal função avaliar aspectos éticos das pesquisas que envolvem seres humanos no Brasil 5 .

A primeira resolução que disparou o processo de criação da CONEP foi a Resolução CNS no 196/19966 (CNS-196). Um grupo executivo de trabalho assumiu as atribuições da CONEP até a sua constituição. Essa resolução inicia-se com um preâmbulo listando vários documentos internacionais que envolvem declarações sobre a pesquisa com humanos, nos quais elencam os princípios éticos que norteiam a resolução. A maioria dos outros artigos da resolução ou retoma esses princípios na forma de diretrizes, ou estabelece normas regulamentadoras que os evidenciam. Nessa resolução, estabelece-se os Comitês de Ética em Pesquisa (CEP) como colegiados interdisciplinares e independentes, tendo como um de seus objetivos defender os interesses dos sujeitos da pesquisa em sua integridade e dignidade.

A Resolução CNS no 466/20127(CNS-466) surge 16 anos depois, revogando a CNS-196. O processo de revisão da CNS-196 ocorreu através de um processo de consulta pública e análise dos participantes do Encontro Nacional dos Comitês de Ética em Pesquisa, recebendo, por fim, o referendo do CNS [Novoa 2014, p. vii]. Os princípios éticos que regiam a CNS-196 permanecem os mesmos nessa resolução, com a incorporação de alguns novos documentos internacionais. Dentre as modificações, destaca-se a inclusão de mais termos e definições, além de alterações em relação ao processo do consentimento livre e esclarecido.

Quatro anos após, o CNS emite mais uma resolução dando atenção especial para pesquisas distintas da área biomédica. O Artigo XIII.3 da CNS-466 já reconhe-

\footnotetext{
${ }^{5}$ Vem em http: / / conselho.saude.gov.br/comissoes-cns/conep/

${ }^{6}$ Ver em https://tinyurl.com/resolucao-cns-196

${ }^{7}$ Ver em https://tinyurl.com/resolucao-cns-466
} 
cia as especificidades éticas existentes das pesquisas nas Ciências Humanas e Sociais. A Resolução CNS no 510/2016 (CNS-510) expandiu esse reconhecimento, possibilitando a apreciação ética de pesquisas científicas que não estivessem condicionadas a conceitos tradicionais de neutralidade e cientificidade tão rígidos. Essa resolução foi um grande avanço para a ciência brasileira, pois criou um conjunto mais adequado de princípios e normas que atendem mais satisfatoriamente à abordagem de pesquisa qualitativa [Guerriero e Minayo 2019, p. 308].

Muitas das pesquisas envolvendo seres humanos na Computação são melhor contempladas nas especificidades descritas na CNS-510 do que na CNS-466. É importante ressaltar que a CNS-510 não invalida e nem revoga a CNS-466, tendo em vista que esta previa aquela em um de seus artigos. Dessa forma, a ética em pesquisa na Computação envolvendo seres humanos deve observar tanto a CNS-466 quanto a CNS-510.

Uma das dificuldades de ordem prática para a observação das resoluções do CNS é a submissão dos projetos de pesquisa para os CEPs. A Comissão Especial de Informática na Educação, por exemplo, publicou um capítulo específico em seu livro de metodologia científica sobre como realizar este processo [Fronza 2020]. Pesquisadores de outras áreas também mencionam dificuldades com o uso da Plataforma Brasil 9 , relatando problemas referentes à usabilidade, levando usuários a duplicar a mesma informação no sistema [Araujo e Francisco 2017, p. 372].

Para pesquisadores da Computação, a origem dessas dificuldades pode ser de uma outra ordem. A discussão sobre a necessidade de se atentar sobre a observância da ética em pesquisa na Computação é relativamente recente. Dessa forma, a dificuldade dos pesquisadores da Computação, sejam docentes ou discentes, deve-se em muito (i) ao desconhecimento da própria discussão em si e, por consequência, (ii) da percepção da importância de se atentar para estas questões de ética em pesquisa.

\section{4. Ética em pesquisa na Computação}

Uma terceira reflexão sobre a formação na ética em pesquisa na Computação envolvendo humanos diz respeito à compreensão dos pressupostos filosóficos e deontológicos existentes na ética em pesquisa atualmente. A ética em pesquisa, de uma forma geral, está assentada em quatro grandes princípios éticos: (i) consentimento livre e esclarecido, (ii) valor científico, (iii) beneficência, (iv) confidencialidade. Esses princípios são explicitamente mencionados na CNS-196 (Artigo III.1) e na CNS-466 (Artigo III.1); e indiretamente mencionados na CNS-510 (Artigo $3^{\circ}$ ).

Singer e Vinson descrevem esses grandes princípios em detalhes, problematizando-os a partir de cenários reais existentes em estudos empíricos na Engenharia de Software [Singer e Vinson 2002, p. 1172]. É importante ressaltar que todas estas problematizações apresentadas para EEE têm uma versão correlata em outras áreas de pesquisa na Computação como EC e IE.

O consentimento livre e esclarecido do participante da pesquisa é um destes grandes princípios. Os elementos fundamentais desse princípio são: (a) a divulgação do propósito da pesquisa ao participante, (b) as condições adequadas para uma devida com-

\footnotetext{
${ }^{8}$ Ver em https: / / tinyurl.com/resolucao-cns-510

${ }^{9}$ Ver em http://plataformabrasil.saude.gov.br/.
} 
preensão da pesquisa, (c) o consentimento (ou não) em participar da pesquisa, e (d) o direito de se retirar da pesquisa em qualquer momento de sua realização. Hundhausen e colegas, ao realizar uma pesquisa sobre o uso de metodologias ativas na EC, solicitaram o consentimento livre e esclarecido dos participantes [Hundhausen et al. 2013, p. 14:9].

O valor científico da pesquisa é mais um grande princípio. Os elementos fundamentais desse princípio são: (a) a importância da pesquisa, e (b) a (in)validade dos resultados. A ACM deixa bem claro em seu Código de Ética ${ }^{1}$, no Art. 2.6, que os seus membros devem trabalhar apenas em áreas de sua competência. Um dos riscos de não se observar esse artigo é a produção de resultados inválidos em uma pesquisa, por exemplo (o que seria eticamente condenável).

Um outro grande princípio é o da beneficência para os participantes da pesquisa. É importante salientar que esse princípio também considera os pesquisadores e as próprias organizações (e.g. universidades, empresas) como usufrutuária dessa potencial beneficência. O princípio em questão balanceia entre os benefícios a todos os envolvidos na pesquisa (incluindo a sociedade como um todo) e os eventuais prejuízos que a pesquisa possa causar. Sieber apresenta os tipos de riscos mais comuns para os participantes da pesquisa em EEE (incluindo o risco relacionado aos pesquisadores) [Sieber 2001].

E, por fim, o último grande princípio é a confidencialidade dos participantes da pesquisa. Os elementos fundamentais desse princípio são: (a) o anonimato e (b) a confidencialidade. Existe o anonimato se a identidade dos participantes da pesquisa é preservada. A confidencialidade é garantida se existe a privacidade dos dados coletados. Silva, em sua dissertação sobre inclusão digital na rede pública de ensino do Distrito Federal, utilizou os codinomes "Sol" (escola de bairro rico) e "Lua" (escola de bairro pobre) para garantir a confidencialidade das escolas no relato da pesquisa [Silva 2019].

Como já mencionado, o desconhecimento da própria discussão da ética em pesquisa na Computação pode ser uma das dificuldades dos pesquisadores da área. Entrevistas realizadas por Fonseca e colegas, sobre como projetar pesquisa qualitativa em EEE, não registraram nenhuma resposta explícita no artigo, por parte dos especialistas entrevistados, referente à necessidade de submissão do projeto de pesquisa a um comitê de ética, por exemplo [Fonseca et al. 2017]. Um levantamento feito por Badampudi mostra que apenas dois artigos (em sete), de um periódico em EEE, mencionaram considerações éticas no corpo do texto do trabalho [Badampudi 2017].

\section{Desafios na Formação sobre o Tema}

Diante do exposto anteriormente, podemos elencar, a seguir, alguns desafios que consideramos importantes sobre a formação na ética em pesquisa na Computação envolvendo humanos

\subsection{Estruturar currículos}

A estruturação de um currículo básico para o tema é bastante importante. Diante dos quatro grande princípios apresentados na Seção 4, seria importante que eles fossem contextualizados e problematizados especificamente para a área de Computação. Deveria existir currículos de formação tanto para o pesquisador que ainda está em formação, quanto para o pesquisador já experiente, para ações específicas de formação continuada. 


\subsection{Construir material didático}

A construção de um material didático é importante para o apoio dos docentes e discentes durante a implementação de um dado currículo. Uma possibilidade interessante é que os livros de metodologia de pesquisa na área de Computação contemple tópicos sobre o tema. Livros de metodologia científica para Computação (e.g. [Wazlawick 2014]) costumam apresentar questões referentes a plágio e direitos autorais, mas não tocam nas questões sensíveis sobre a ética envolvendo humanos.

\subsection{Incluir exigência em congressos e periódicos}

Uma das formas de se exigir uma mudança de postura da comunidade científica é elevar o padrão nos principais meios de publicação: congressos e periódicos. Reconhecer a importância da ética em pesquisa na Computação passa pelo reconhecimento da necessidade desses meios exigirem tais aspectos. Alguns periódicos em áreas da Educação no Brasil, por exemplo, exigem que as submissões mencionem explicitamente a aprovação obtida por meio de algum CEP (e.g. Revista Educação Especial ${ }^{10}$ ). Entretanto, a maioria dos periódicos e das trilhas científicas em eventos da Computação não costumam apresentar essa exigência. Como primeiro passo, poderia-se exigir a menção nos artigos de como foram tratadas as questões éticas que naturalmente emergem durante a realização da pesquisa envolvendo seres humanos.

\subsection{Promover iniciativas educativas}

É necessário que todos os envolvidos na condução de um projeto de pesquisa recebam formação adequada sobre o tema. Instituições, gestores, órgãos de fomento / financiadores e participantes (sujeitos) da pesquisa apresenta o grande leque de possibilidades que existem nessas iniciativas.

Cotler e colegas, por exemplo, promoveram uma intervenção educacional híbrida [Cotler et al. 2015, p. 83]. A proposta ocorreu dentro de um curso de verão que investigava questões de cyberbulling em ambientes de jogos online. Entretanto, simultaneamente, foi solicitado aos estudantes a realização de um processo semelhante à submissão de um projeto para um CEP.

\subsection{Atuar politicamente}

Uma das percepções errôneas sobre a ética em pesquisa é que ela deve ser apenas observada de maneira passiva. É importante que sejam apresentados os caminhos já existentes para que propostas de modificação sejam encaminhadas e, possivelmente, incorporadas à atual legislação de ética em pesquisa. A ética em pesquisa no Brasil precisa ser continuamente evoluída (como apresentada historicamente na Seção 3) de forma que a Computação pode (e deve) contribuir para que as resoluções atuais reflitam e promovam os desafios sobre o tema em relação à nossa área.

Um dos meios já existentes é que mais membros da Computação participem ativamente dos CEPs mais próximos de sua instituição [Munteanu et al. 2015, p. 112]. Essa participação pode ser como membro do próprio CEP ou através de diálogos com os membros dos CEPs. Assim, ao apresentar as particularidades de nossa área, podemos propor a criação de protocolos que promovam a ética em pesquisa na Computação, incentivando também um número maior de submissões nos respectivos CEPs. É necessário que

\footnotetext{
${ }^{10}$ Ver em http://gg.gg/rev-educacao-especial-cep.
} 
a própria comunidade científica regule a atuação dos CEPs e da CONEPE para que eventualmente, por meio de uma compreensão equivocada de algum aspecto de pesquisa na Computação, não proíba ou permita equivocadamente algum projeto ou etapa da pesquisa.

\subsection{Ampliar o escopo de disciplinas}

Em alguns currículos de Computação, a ética em pesquisa costuma ser contemplada em disciplinas de metodologia científica, direito ou filosofia. Entretanto, é bastante comum que os tópicos abordados nessas disciplinas se restrinjam à legislação, propriedade intelectual, questões de autoria, relação orientador/aluno e fraude científica.

Uma das formas de potencializar a presença do tema em nossos currículos é ampliar o escopo dessas disciplinas para incluir, por exemplo, tópicos envolvendo um dos quatro grandes princípios apresentados na Seção 4 . Dessa forma, os próprios núcleos docentes estruturantes poderiam agir de maneira mais efetiva nessa frente, através de adequação do ementário de disciplinas ou até por meio de uma reformulação do próprio projeto pedagógico do curso. Alguns cursos contemplam tópicos de ética na Computação de forma transversal, perpassando as disciplinas da estrutura curricular (e.g. [Reich et al. 2020]). Esse seria um caminho interessante para promover mudanças significativas dentro da própria instituição de ensino.

\subsection{Promover os referenciais de formação da SBC}

Em uma dimensão nacional dessa agenda, uma possibilidade seria a promoção do que já está disposto nos referenciais de formação da SBC. Por exemplo, os Referenciais de Formação para os Cursos de Pós-graduação Stricto Sensu em Computação [Araujo et al. 2019, p. 9] propõe um eixo de formação em ética, evidenciando a necessidade de se perseguir as boas práticas de pesquisa. É proposto como uma das competências derivadas a ser desenvolvida na formação do pesquisador que ele avalie "os impactos de seu trabalho nas organizações, na sociedade e no meio ambiente, considerando o desdobramento de seus resultados".

\section{Considerações Finais}

Esse ensaio teve como objetivo trazer reflexões e desafios sobre a formação na ética em pesquisa na Computação envolvendo humanos. O tema foi apresentado tanto no contexto geral, como no contexto específico da Computação.

É importante ressaltar que o propósito desse ensaio não foi fornecer uma perspectiva "moralista" ou impositiva sobre como devemos formar os pesquisadores em Computação que fazem suas investigações envolvendo participantes humanos. $\mathrm{O}$ propósito dos autores é contribuir para que as respostas para a pergunta "Devo ou não submeter o meu projeto de pesquisa para o Comitê de Ética? [Amorim et al. 2019]" sejam estruturadas de uma forma mais qualificada, com a reflexão adequada que esse tema merece. E, para isso, a promoção da formação sobre o tema é necessária.

\section{Agradecimentos}

O presente trabalho foi realizado com apoio da Coordenação de Aperfeiçoamento de Pessoal de Nível Superior - Brasil (CAPES). 


\section{Referências}

Amorim, P. F., Sacramento, C., Capra, E. P., Tavares, P. Z., e Ferreira, S. B. L. (2019). Submit or not my HCI research project to the ethics committee, that is the question. In Proceedings of the 18th Brazilian Symposium on Human Factors in Computing Systems, IHC '19, New York, NY, USA. Association for Computing Machinery.

Araujo, N. e Francisco, D. (2017). Ética em pesquisa com seres humanos na web: o caso da Plataforma Brasil. Informação \& Informação, 21(3):361-375.

Araujo, R., Simão, A., Malucelli, A., Zorzo, A., Monteiro, J. A., e Chaimowicz, L. (2019). Referenciais de formação para os cursos de pós-graduação Stricto Sensu em Computação 2019. Sociedade Brasileira de Computação.

Badampudi, D. (2017). Reporting Ethics Considerations in Software Engineering Publications. In 2017 ACM/IEEE International Symposium on Empirical Software Engineering and Measurement (ESEM), pages 205-210.

Brey, P. e Søraker, J. H. (2009). Philosophy of Computing and Information Technology. In Meijers, A., editor, Philosophy of Technology and Engineering Sciences, Handbook of the Philosophy of Science, pages 1341 - 1407. North-Holland, Amsterdam.

Buchanan, E., Aycock, J., Dexter, S., Dittrich, D., e Hvizdak, E. (2011). Computer science security research and human subjects: Emerging considerations for research ethics boards. Journal of Empirical Research on Human Research Ethics, 6(2):71-83.

Cooper, S., Dann, W., e Pausch, R. (2003). Teaching Objects-First in Introductory Computer Science. SIGCSE Bull., 35(1):191-195.

Cotler, J. L., Fryling, M., Rivituso, J., Mathews, L., e Pratico, S. (2015). The social side of cs: engaging undergraduate computer science students in the irb process for research investigating cyberbullying in online multi-player gaming environments. Journal of Computing Sciences in Colleges, 30(6):82-88.

Easterbrook, S., Singer, J., Storey, M.-A., e Damian, D. (2008). Selecting Empirical Methods for Software Engineering Research, pages 285-311. Springer London, London.

Fonseca, L. S. S., Seaman, C. B., e Soares, S. C. B. (2017). Describing What Experimental Software Engineering Experts Do When They Design Their Experiments - A Qualitative Study. In 2017 ACM/IEEE International Symposium on Empirical Software Engineering and Measurement (ESEM), pages 211-216.

Fronza, C. A. (2020). Submissão de projeto de pesquisa ao Comitê de Ética: da Plataforma Brasil ao Parecer Consubstanciado. In Jaques, P. A., Pimentel, M., Siqueira, S., e Bittencourt, I., editors, Metodologia de Pesquisa Científica em Informática na Educação: Concepção da Pesquisa, volume 1 of Série Metodologia de Pesquisa em Informática na Educação. SBC, Porto Alegre.

Guerriero, I. C. Z. e Minayo, M. C. (2019). A aprovação da Resolução CNS nº 510/2016 é um avanço para a ciência brasileira. Saúde e Sociedade, 28:299-310.

Hundhausen, C. D., Agrawal, A., e Agarwal, P. (2013). Talking about Code: Integrating Pedagogical Code Reviews into Early Computing Courses. ACM Trans. Comput. Educ., 13(3). 
Johnson, D. G. (2008). Computer Ethics, chapter 5, pages 63-75. John Wiley \& Sons, Ltd.

Kizza, J. M. (2016). Ethics in Computing. Springer.

Kunkle, W. M. e Allen, R. B. (2016). The impact of different teaching approaches and languages on student learning of introductory programming concepts. ACM Trans. Comput. Educ., 16(1).

Masiero, P. C. (2000). Ética em computação. Edusp.

Munteanu, C., Molyneaux, H., Moncur, W., Romero, M., O’Donnell, S., e Vines, J. (2015). Situational ethics: Re-thinking approaches to formal ethics requirements for human-computer interaction. In Proceedings of the 33rd Annual ACM Conference on Human Factors in Computing Systems, pages 105-114.

Noelliste, M. (2013). Integrity: An intrapersonal perspective. Human Resource Development Review, 12(4):474-499.

Novoa, P. C. R. (2014). O que muda na ética em pesquisa no Brasil: Resolução 466/12 do Conselho Nacional de Saúde. Einstein (São Paulo), 12(1):vii-vix.

Rallis, S. F. e Lawrence, R. B. (2017). Integrity Is More Than Validity: Seeking Credible, Usable, and Ethical Research. New Directions for Institutional Research, 2017(174):25-38.

Reich, R., Sahami, M., Weinstein, J. M., e Cohen, H. (2020). Teaching computer ethics: A deeply multidisciplinary approach. In Proceedings of the 51st ACM Technical Symposium on Computer Science Education, pages 296-302.

Saltz, J., Skirpan, M., Fiesler, C., Gorelick, M., Yeh, T., Heckman, R., Dewar, N., e Beard, N. (2019). Integrating Ethics within Machine Learning Courses. ACM Trans. Comput. Educ., 19(4).

Sieber, J. E. (2001). Protecting research subjects, employees and researchers: Implications for software engineering. Empirical Software Engineering, 6(4):329-341.

Silva, C. C. G. (2019). Inclusão digital na educação básica: comparando duas escolas públicas dos anos iniciais do Distrito Federal. Master's thesis, Universidade Católica de Brasília (UCB), Brasília.

Singer, J. e Vinson, N. G. (2002). Ethical issues in empirical studies of software engineering. IEEE Transactions on Software Engineering, 28(12):1171-1180.

Stahl, B. C. (2019). Teaching ethical reflexivity in information systems: How to equip students to deal with moral and ethical issues of emerging information and communication technologies. Journal of Information Systems Education, 22(3):8.

Tedre, M. (2007). Know your discipline: Teaching the philosophy of computer science. Journal of Information Technology Education: Research, 6(1):105-122.

Vinson, N. G. e Singer, J. (2008). A practical guide to ethical research involving humans. In Guide to Advanced Empirical Software Engineering, pages 229-256. Springer.

Wazlawick, R. (2014). Metodologia de pesquisa para Ciência da Computação. Elsevier Brasil, 2 edition. 